\title{
“Eli-P-Complex" Diagnostic Test for Preconception Care in Women with History of Adverse Pregnancy Outcome: A Randomized Multicenter Trial
}

\author{
Svetlana G. Tsakhilova ${ }^{1}$, Tatiana E. Sharkovskaya², Olga A. Yakimovich ${ }^{3}$, \\ Aida M. Begizova1, Angelina A. Malsagova1 \\ ${ }^{1}$ Moscow State University of Medicine and Dentistry, Maternity Hospital № 8, Moscow, Russia \\ ${ }^{2}$ Family Planning Center, Rostov-on-Don, Russia \\ ${ }^{3}$ Medical Institute "REAVIZ", Moscow, Russia \\ Email: olya.yakimovich@gmail.com
}

Received 1 September 2015; accepted 3 November 2015; published 6 November 2015

Copyright (C) 2015 by authors and Scientific Research Publishing Inc.

This work is licensed under the Creative Commons Attribution International License (CC BY). http://creativecommons.org/licenses/by/4.0/

(c) (i) Open Access

\section{Abstract}

Background: ELI-P-Complex is the most advanced diagnostic test designed to assess whether the female body is ready (or unready) for the normal pregnancy course and for giving birth to a healthy child. ELI-P-Complex enables the perceived abnormalities to be individually treated even prior to the pregnancy planning, thus minimizing the risk of gestational and delivery-related complications. This prospective study shows the effectiveness of ELI-P-Complex testing during preconception care in women with a history of adverse pregnancy outcomes (APO). Methods: The data were reviewed from 4519 women with a history of APO and who planned to get pregnant. Following randomization, subjects of Group A were tested with ELI-P-Complex and treated before the pregnancy based on the results obtained. Group B subjects were not examined using the ELI$P$-Complex test and were prepared for pregnancy in accordance with the standard strategy of preconception care [1]. Results: In Group A, gestational complications (GC) were revealed in $20 \%$ of women compared to $88.7 \%$ of Group B subjects; the relative risk (RR) of GC was 11.67 (95\% CI: 9.9851 to 13.6392; $P<0.0001$ ). APO was reported in $12 \%$ of Group A subjects vs. $38.1 \%$ of Group B ones; RR of APO was 5.8908 (95\% CI: 4.9365 to 7.0296; $\mathrm{P}<0.0001)$. Absolutely healthy children were born from $88.1 \%$ of Group A subjects compared to $50.3 \%$ of Group B ones; RR was 7.9601 ( $95 \%$ CI: 6.6110 to $9.5845 ; \mathrm{P}<0.0001$ ). The positive predictive value, sensitivity, and specificity of the test for GC were $93.82 \%$ (95\% CI: $92.72 \%$ to $94.80 \%), 93.17 \%$ (95\% CI: $92.03 \%$ to $94.20 \%$ ), and $92.62 \%$ (95\% CI: $\mathbf{9 1 . 3 1 \%}$ to $93.78 \%$ ), respectively. Conclusions: The use of ELI-P-Complex for 
examination and further preconception care in women with a history of APO considerably reduces GC, improves pregnancy outcomes, and increases chances of giving birth to a healthy child.

\title{
Keywords
}

\author{
APO-Adverse Pregnancy Outcomes, GC-Gestational Complications, RR-Relative Risk
}

\section{Introduction}

Adverse pregnancy outcomes (APO), primarily including miscarriages, intrauterine fetal death, and giving birth to children with developmental malformations, are observed rather often. Miscarriage affects at least $10 \%$ of couples trying to conceive, while one in every 33 babies in the United States is born with a birth defect [2]. The couples experiencing an adverse pregnancy outcome may be at risk of having other adverse outcomes in future pregnancies. Recurrent miscarriage affects $1 \%$ of couples trying to conceive [3]. Based on Regan et al.'s [4] data, National Institute for Health and Care Excellence (NICE, UK) states that after each miscarriage the possibility of recurrent miscarriage increases by $20 \%$ [NICE]. The recurrence risk for stillbirth increases twofold to tenfold in the next pregnancy [5] [6]. In addition, one type of adverse pregnancy outcome can also increase the risk of other adverse pregnancy outcomes [7].

It is known that only a small part of APO (5\% - 13\%) is caused by genetic aberrations, while most birth defects and miscarriages are associated with epigenetic developmental disorders [8]-[10]. It is almost impossible to find out the cause of these disorders (despite a large number of laboratory tests and diagnostic procedures requiring a lot of time and material resources). However, it is well known that very different environmental factors (an excess of heavy metals, organic pollutants, viruses, etc.) adversely affecting the body of a pregnant woman can change the state of her immune system. On the other hand, disturbances in immune regulation, resulting in surplus or insufficient production of natural embryotropic auto-antibodies (a-Abs) of the IgG class that negatively influences the gestation process, are one of the most common reasons of adverse pregnancy outcome [11]-[13]. Usually, serum contents of various a-Abs in healthy women are maintained within relatively narrow concentration ranges [14]. In contrast, in $75 \%-95 \%$ of women suffering from habitual miscarriages or other forms of pregnancy complications, long-lasting abnormalities of a-Abs may be found [14]. Due to multiple mechanisms of protection and compensation, immune deviations do not necessarily lead to poor pregnancy outcomes, but sharply increase the probability of miscarriages, fetal deaths, or developmental malformations [14][17]. Another important issue is the influence of maternal immune deviations upon the health of the child. Changes in a-Abs contents almost always negatively influence the health state of a newborn [11]. Therefore, by analyzing some parameters of a female immune system, we can predict the course of a planned pregnancy, on the one hand, and on the other hand, come to better understanding of which external factors cause immune disorders and try to eliminate these factors prior to pregnancy planning [9].

Recently a laboratory diagnostic test called ELI-P-Complex [18] has been used to predict the course and outcome of the pregnancy. It is able to assess the state of the female reproductive system with just a single analysis and give a clear understanding of the cause of disorder, therefore turning the diagnostic search in the right direction that prevents spending money on other blindly conducted studies [18]-[20]. In our prospective study, we have investigated the possibilities of using ELI-P-Complex method for preconception care in women with a history of adverse pregnancy outcomes in order to improve outcomes of the subsequent pregnancies.

\section{Methods}

The study was conducted from April 2008 until June 2014 (the last participant was enrolled in March 2013). Initially, 4519 female patients aged 17 to 45 years were enrolled for its conduction; they visited special family planning centers in Moscow, Saint-Petersburg, Kazan, Novosibirsk, and Rostov-on-Don (Russian Federation). Later, all study participants were followed up in out-patient service at Obstetrics and Gynecology Departments in nineteen hospitals of the above-mentioned cities. For delivery, as well as in case of any pregnancy complications, all participants were mandatorily hospitalized in these particular hospitals.

The main criterion for inclusion in the study was the presence in the medical history at least one of the fol- 
lowing adverse pregnancy outcomes: miscarriage, missed miscarriage, anembryonic pregnancy (blighted ovum), fetal death (stillbirth), intrauterine growth restriction, premature birth, and fetal and congenital malformations. Fertile women without a history of adverse pregnancy outcomes, as well as women diagnosed with infertility, were withdrawn from the study. The study was approved by the research ethics committees at all participating hospitals, and women gave informed consent before enrolling in the study.

Women who were eligible for the study were randomly allocated to two groups: the group of preconception care based on ELI-P-Complex testing data (Group A, $n=2209$ ) and the group of preconception care without ELI-P-Complex testing (Group B, $\mathrm{n}=2310$ ) (Table 1).

Blood serum samples were drawn from all women in Group A prior to the pregnancy for testing with ELI-P-Complex. Freshly obtained blood serum samples stored for not longer than 3 days at a temperature $=$ $+1^{\circ} \mathrm{C} \cdots+4^{\circ} \mathrm{C}$ were used in the study. Analysis was conducted using ELI-P-Complex kits (Immunculus, Moscow).

ELI-P-Complex method. ELI-P-Complex method and kit are intended for measuring the profiles of specific serum immune reactivity (IR) which depends on a serum content of twelve IgG a-Abs [18] [21] (namely a-Abs interacting with Chorionic Gonadotropin (hCG), dsDNA, $\beta 2$-Glycoprotein I, Fc-fragment of IgG (rheumatoid factor), collagen II, insulin, thyroglobulin, proteins of S100 family, Spr-06-antigen of sperm membranes, TrM-03-antigen of platelet membranes, ANCA, KiM-05-antigen of kidney cell membranes. Abnormalities in the content of the above-mentioned a-Abs may prominently influence pregnancy development and fetus formation if their serum content has deviated from normal parameters. The ELI-P-Complex test is registered by the Russian Ministry of Health for diagnostic and prognostic investigation of women planning pregnancy or already pregnant [11].

Median individual immune reactivity (MIR) and profiles of individual immune reactivity (relative content) of the corresponding auto-antibodies were detected and analyzed in the blood serum samples.

Reactions were run according to the manufacturer's instructions using standard procedures of enzyme-linked immunosorbent assay. The optical density level of the immunosorbent reaction of the control serum (CS) with each antigen was considered as $100 \%$, and the intensity of the patients' serums reactions with the same antigens was calculated relative to the CS reaction [22]. Then, the median individual level of immune reactivity of the tested blood serum samples with each antigen was calculated relative to the CS reaction using the following formulas:

$$
\operatorname{MIR}=\left(\frac{R(\operatorname{ag} 1) \cdot 100}{R(k 1)}-100+\frac{R(\operatorname{ag} 2) \cdot 100}{R(k 2)}-100+\cdots+\frac{R(\operatorname{ag} 12) \cdot 100}{R(k 12)}-100\right): 12 \quad \text { [22] [23], }
$$

Abbreviations: MIR — median individual level of immune reactivity of the individual patient's serum regarding

Table 1. Base-line characteristics of a and b groups.

\begin{tabular}{|c|c|c|c|}
\hline Characteristic & Group A $(n=2209)$ & Group B $(n=2310)$ & $\mathbf{P}$ \\
\hline \multicolumn{4}{|l|}{ Maternal age (years) } \\
\hline$\geq 30$ & $1489(67.4 \%)$ & $1483(64.2 \%)$ & $\mathrm{P}<0.05$ \\
\hline$<30$ & $720(32.6 \%)$ & $827(35.8 \%)$ & $\mathrm{P}<0.05$ \\
\hline $\begin{array}{l}\text { Gynecological diseases in the } \\
\text { past medical history }\end{array}$ & $1953(88.4 \%)$ & $2107(91.2 \%)$ & $\mathrm{P}<0.05$ \\
\hline \multicolumn{4}{|l|}{ Outcomes of previous pregnancies } \\
\hline Full term birth & $528(23.9 \%)$ & $566(24.5 \%)$ & $\mathrm{P}>0.05$ \\
\hline Premature birth & $60(2.7 \%)$ & $51(2.2 \%)$ & $\mathrm{P}>0.05$ \\
\hline Fetal and congenital malformations & $82(3.7 \%)$ & $67(2.9 \%)$ & $\mathrm{P}>0.05$ \\
\hline Fetal death (stillbirth) & $53(2.4 \%)$ & $62(2.7 \%)$ & $\mathrm{P}>0.05$ \\
\hline Induced abortion & $371(16.8 \%)$ & $418(18.1 \%)$ & $\mathrm{P}>0.05$ \\
\hline \multicolumn{4}{|l|}{ Miscarriage } \\
\hline 1 & $581(26.3 \%)$ & $621(26.9 \%)$ & $\mathrm{P}>0.05$ \\
\hline 2 & $985(44.6 \%)$ & $1088(47.1 \%)$ & $\mathrm{P}>0.05$ \\
\hline 3 and more & $596(27 \%)$ & $573(24.8 \%)$ & $\mathrm{P}>0.05$ \\
\hline Missed miscarriage & $400(18.1 \%)$ & $411(17.8 \%)$ & $\mathrm{P}>0.05$ \\
\hline Anembryonic pregnancy (blighted ovum) & $75(3.4 \%)$ & $90(3.9 \%)$ & $\mathrm{P}>0.05$ \\
\hline Intrauterine growth restriction & $176(8 \%)$ & $166(7.2 \%)$ & $\mathrm{P}>0.05$ \\
\hline
\end{tabular}


all the used antigens. The normal (physiological) levels of individual MIR are restricted by the ranges $-30 \% \ldots$ to $0 \%$ (or conditional units (CU)) of the control sample median IR.

Moreover, in order to analyze immune reactivity profiles of the studied patients, a deviation (as a percentage of the individual median normalized reaction level) detected in the serum of a studied patient was calculated for each of the antigens using the following formulas:

$$
\begin{aligned}
& R(n r m) \operatorname{ag} 1=\left(\frac{O D(\operatorname{ag} 1) \cdot 100}{O D(k 1)}\right)-100-\mathrm{MIR} ; \\
& R(n r m) \operatorname{ag} 2=\left(\frac{O D(\operatorname{ag} 2) \cdot 100}{O D(k 2)}\right)-100-\mathrm{MIR} ; \cdots ;[22][23] ; \\
& R(\text { nrm }) \operatorname{ag} 12=\left(\frac{O D(\operatorname{ag} 12) \cdot 100}{O D(k 12)}\right)-100-\mathrm{MIR}
\end{aligned}
$$

Abbreviations: $R(\mathrm{nrm}) \mathrm{ag} 1, \mathrm{ag} 2, \cdots, \mathrm{ag} 12$ - deviation (in fractions of the individual median normalized reaction level) detected in the individual patient's serum for each of the used antigens $-1,-2, \cdots-12$; OD (ag1, ag2, $\cdots$ ag12) - optic density of the individual patient's serum reaction with each of the used antigens $-1,-2, \cdots-12$; $\mathrm{OD}(\mathrm{k} 1, \mathrm{k} 2 \cdots \mathrm{k} 12)$ - optic density of the CS with each of the used antigens $-1,-2, \cdots-12$. The normal (physiological) level of each $\mathrm{a}-\mathrm{Ab}$ is restricted by the ranges $-20 \% \cdots$ to $10 \%$ (or conditional units (CU) from the individual average IR). A special computer program supplied together with the kits was used for calculations.

In Group A, disorders revealed with ELI-P-Complex test were confirmed by routine methods of diagnostics according to the unified criteria for patient examination recommended after ELI-P-Complex testing. Treatment of detected disorders was conducted individually in accordance with the common methods of disease treatment. In 3 - 4 weeks after the treatment completion, a control examination was conducted using ELI-P-Complex test. In this study, absolutely all women were allowed to plan pregnancy regardless of the test result solely for scientific purposes; however, the manufacturer of ELI-P-Complex test recommends pregnancy planning strictly according to the following criteria: 1) normalization of the serum content of embryotropic a-Abs; 2) decrease in initially elevated level of the immune reactivity of certain a-Abs by at least $50 \%$ from baseline; 3 ) increase in median individual reactivity (MIR) level of at least 1.5 fold the initial value in women with immune suppression at baseline; and 4) decrease in the median individual reactivity (MIR) level to the normal values in women with initial immune activation. Absence of the above-mentioned changes indicates that the treatment is not effective and a specific pathogenic cause persists which can result in recurrence of the adverse pregnancy outcome.

Pregnancy onset in Group A was registered 3 - 9 months after the first investigation with ELI-P-Complex test. In total, pregnancy was diagnosed in 1971 women from Group A. In 238 cases pregnancy did not happen during the 6-month period of observation, and such patients were excluded from the study. In Group B, preconception care was not conducted using ELI-P-Complex test, and the women started pregnancy planning right after randomization. In Group B, pregnancy was confirmed in 1998 women. In the remaining 312 women, pregnancy did not occur within 6 months and they were withdrawn from the study.

In both groups (Group A and Group B), ELI-P-Complex test was performed during the first, second, and third trimester, as well as all standard laboratory tests, ultrasound scanning to assess the pregnancy course, the condition of mother and child, and also the condition of a newborn right after the delivery. Clinical monitoring of the pregnancy course was initiated at weeks 6 - 9 of gestation and was continued until its completion. Health state of children delivered by women under observation was assessed within the first $5-7$ days after birth (Figure 1).

\section{Statistical Analysis}

All statistical analyses were performed using the SPSS 21.0.0 for Windows (IBM, NY, USA) and STATA/SE 13.0 software (STATA Corp., Texas, USA) taking into consideration $95 \%$ CI. The chi-square test was used to determine significant differences. Performance indicators (positive predictive value (PPV), sensitivity, specificity) of ELI-P-Complex test have been calculated for pregnancy complications. Relative risk of an adverse pregnancy outcome and delivery of an unhealthy child was calculated by comparing Group A (with preconception care with ELI-P-Complex testing) and Group B (without ELI-P-Complex testing during the preconception care). 


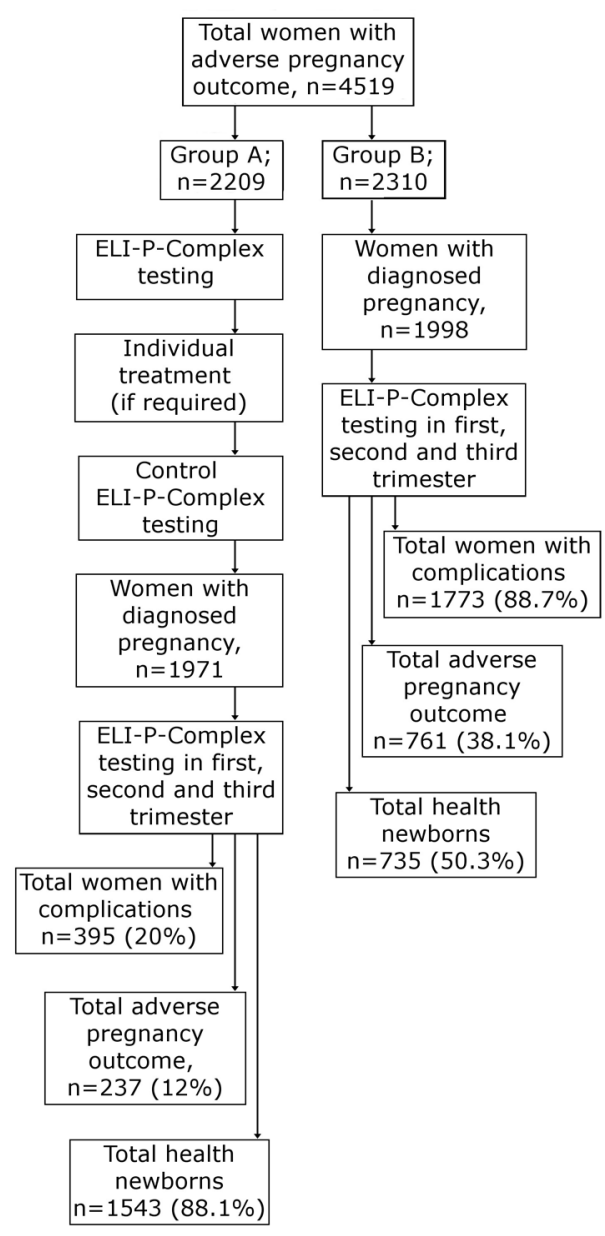

Figure 1. Study flow diagram.

\section{Results}

Initially almost 2209 non-pregnant women with a history of adverse pregnancy outcomes were tested using ELI-P-Complex (Group A). It was found that disorders of serum immune reactivity determined by natural IgG auto-antibodies are revealed in more than $90 \%$ of such women: abnormalities were reported in $92.4 \%(n=2041)$ of them, and only in $7.6 \%(n=168)$ of patients ELI-P-Complex test scores were within the normal range. The changes revealed included abnormal decrease or, vice versa, abnormal increase in total immune reactivity level in the blood serum with all or with the most of the used antigens (abnormalities of the median individual immune reactivity, MIR). Furthermore, abnormal changes could selectively relate to separate a-Abs of certain specificity.

Additional clarifying investigations prescribed based on screening ELI-P-Complex data made it possible to specify in detail the situation in each case. In $83.5 \%(n=1704)$ of women from Group A, abnormal increases in microbial population potentially important for adverse pregnancy outcome (including chlamydia, mycoplasma, ureaplasma, gardnerella, trichomonasvaginalis, candida, and herpes viruses) was detected. In $65.6 \%(n=1339)$ of women, hormonal disorders of various grades were revealed (thyroid disorders, disorders of menstrual cycle, polycystic ovary syndrome, hyperandrogenism, hyperprolactinaemia, hypogonadism, hypoprogesteronaemia, and obesity). In $8.2 \%(n=167)$ of cases, the signs of pancreatitis were revealed; notably, in almost a quarter of such women $(n=38)$ the signs of glucose intolerance and/or metabolic syndrome were observed. In some of the examined women, the signs of antiphospholipid syndrome $(18.6 \%, \mathrm{n}=380)$ were present; small vessels vasculitis $(12.1 \%, n=245)$, thrombocytopathy $(9.1 \%, n=186)$, and changes in hemostasis $(8.3 \%, n=169)$ were revealed in nearly one third of patients. An abnormally elevated level of antibodies against chorionic gonadotropin was detected in $3.4 \%$ of women $(n=69)$, pituitary adenoma was revealed in $2(0.1 \%)$, and the signs of prema- 
ture ovarian aging were observed in $9(0.44 \%)$ of women. The signs of papillomavirus infection were revealed quite often (in $24.4 \%, \mathrm{n}=498$ ) which is a rather common cause of pathologic pregnancy. Rather often $(16.8 \%, \mathrm{n}=$ 343 ) the signs of endometritis and/or inflammatory processes in other pelvic organs were present. Finally, in 5.4\% $(\mathrm{n}=110)$ of cases, chronic pyelonephritis was diagnosed.

Based on the results of clarifying investigations, individual treatment was prescribed. After treatment completion, control ELI-P-Complex testing revealed full normalization of test parameters in $35.9 \%(\mathrm{n}=733)$ of women and improvement of the scores up to the values recommended for pregnancy planning in $47 \%$ (959) of patients. In $17.1 \%$ of cases (349 women), no clear improvement of ELI-P-Complex test scores was achieved.

Women from Group B $(n=2310)$ were not examined using ELI-P-Complex method prior to the pregnancy, and their care was conducted according to the uniform common regimens of preconception care, recommended by NICE [1].

In the first, second, and third trimesters of pregnancy, all Group A patients underwent additional ELI-PComplex testing. In a majority of cases $(94.4 \%)$, test scores fluctuated only slightly $( \pm 12 \%)$ compared to the values reported after preconception care shortly before pregnancy onset. Only $3.7 \%(n=73)$ of pregnant women experienced significant $(>12 \%)$ changes in the immune reactivity parameters; in $63(3.2 \%)$ of them, changes were transient and were detected during acute respiratory diseases.

After onset of pregnancy, the patients from Group B also underwent ELI-P-Complex testing during the first, second, and third trimesters. Deviations of ELI-P-Complex test scores in Group B women were similar to those observed in Group A women prior to their preconception care. Only 130 (6.5\%) pregnant women from Group B had normal ELI-P-Complex test scores on the first examination (in the first trimester of pregnancy), while abnormal decrease/increase in total immune reactivity level or abnormal changes of a-Abs of certain specificity were detected in $93.5 \%(\mathrm{n}=1868)$ of cases. During the second trimester, normalparameters were observed already in $16.2 \%$ of Group B women, and in $24.4 \%$ during the third trimester. However, positive changes in ELIP-Complex test scores over time during the pregnancy course are relative and most possibly are related to the sequential drop-out of women (pregnancy loss) whose reproductive health was especially poor. Furthermore, therapeutic measures conducted for certain complications affected the improvement of ELI-P-Complex test scores as well.

Summary data concerning peculiarities of pregnancy course and condition of the newborns delivered by women from groups A and B are presented in Tables 2-4.

Table 2. Complications of the pregnancy course.

\begin{tabular}{|c|c|c|c|}
\hline Pregnancy complications & Group A $(n=1971)$ & Group B $(n=1998)$ & $\mathbf{P}$ \\
\hline \multicolumn{4}{|c|}{ First trimester } \\
\hline Threatened miscarriage & $150(7.6 \%)$ & $1746(87.4 \%)$ & $\mathrm{P}<0.01$ \\
\hline Cervical incompetence & $63(3.2 \%)$ & $116(5.8 \%)$ & $\mathrm{P}<0.01$ \\
\hline \multicolumn{4}{|c|}{ Second trimester } \\
\hline Threatened miscarriage & $71(3.6 \%)$ & $957(47.9 \%)$ & $\mathrm{P}<0.01$ \\
\hline Pre-eclampsia & $51(2.6 \%)$ & $106(5.3 \%)$ & $\mathrm{P}<0.01$ \\
\hline Placentitis & $16(0.8 \%)$ & $144(7.2 \%)$ & $\mathrm{P}<0.01$ \\
\hline Intrauterine hypoxia & $73(3.7 \%)$ & $703(35.2 \%)$ & $\mathrm{P}<0.01$ \\
\hline \multicolumn{4}{|c|}{ Third trimester } \\
\hline Threatened premature labor & $34(1.7 \%)$ & $537(26.9 \%)$ & $\mathrm{P}<0.01$ \\
\hline Pre-eclampsia & $71(3.6 \%)$ & $204(10.2 \%)$ & $\mathrm{P}<0.01$ \\
\hline Fetal malformation & - & $10(0.5 \%)$ & $\mathrm{P}<0.01$ \\
\hline Intrauterine growth restriction & $55(2.8 \%)$ & $186(9.3 \%)$ & $\mathrm{P}<0.01$ \\
\hline Intrauterine hypoxia & $93(4.7 \%)$ & $430(21.5 \%)$ & $\mathrm{P}<0.01$ \\
\hline Polyhydramnios & $18(0.9 \%)$ & $44(2.2 \%)$ & $\mathrm{P}<0.01$ \\
\hline Oligohydramnios & $37(1.9 \%)$ & $56(2.8 \%)$ & $\mathrm{P}>0.05$ \\
\hline Total pregnant women with complications & $395(20 \%)$ & $1773(88.7 \%)$ & $P<0.01$ \\
\hline
\end{tabular}


Table 3. Pregnancy outcome.

\begin{tabular}{|c|c|c|c|}
\hline Pregnancy outcome & Group A $(n=1971)$ & Group B $(n=1998)$ & $\mathbf{P}$ \\
\hline Full term birth & $1734(88 \%)$ & $1237(61.9 \%)$ & $\mathrm{P}<0.01$ \\
\hline Premature birth & $17(0.9 \%)$ & $224(11.2 \%)$ & $\mathrm{P}<0.01$ \\
\hline Miscarriage in the first trimester & $169(8.6 \%)$ & $327(16.4 \%)$ & $\mathrm{P}<0.01$ \\
\hline Miscarriage in the second trimester & $32(1.6 \%)$ & $112(5.6 \%)$ & $\mathrm{P}<0.01$ \\
\hline Fetal death (stillbirth) & - & $44(2.2 \%)$ & $\mathrm{P}<0.01$ \\
\hline Missed miscarriage & $7(0.3 \%)$ & $28(1.4 \%)$ & $\mathrm{P}<0.01$ \\
\hline Anembryonic pregnancy (blighted ovum) & $12(0.6 \%)$ & $26(1.3 \%)$ & $\mathrm{P}<0.05$ \\
\hline Total adverse pregnancy outcomes & $237(12 \%)$ & $761(38.1 \%)$ & $\mathrm{P}<0.01$ \\
\hline
\end{tabular}

Table 4. Health state of the newborns.

\begin{tabular}{cccc}
\hline Newborn's health state & Group A $(\mathbf{n}=\mathbf{1 7 5 1})$ & Group B (n= 1461) & P \\
\hline Normal (Apgar score $\geq 7)$ & $1543(88.1 \%)$ & $735(50.3 \%)$ & $\mathrm{P}<0.01$ \\
Mild perinatal asphyxia & $106(6.1 \%)$ & $279(19.1 \%)$ & $\mathrm{P}<0.01$ \\
Severe perinatal asphyxia & $13(0.7 \%)$ & $27(1.8 \%)$ & $\mathrm{P}<0.01$ \\
Intrauterine growth restriction & $66(3.8 \%)$ & $118(8.1 \%)$ & $\mathrm{P}<0.01$ \\
Premature infant (low birth weight) & $17(1 \%)$ & $224(15.3 \%)$ & $\mathrm{P}<0.01$ \\
Congenital malformations & $6(0.3 \%)$ & $74(5.1 \%)$ & $\mathrm{P}<0.01$ \\
Early death cases (at the first 2 wks) & - & $4(0.3 \%)$ & $\mathrm{P}<0.05$ \\
Total healthy newborns & $\mathbf{1 5 4 3}(\mathbf{8 8 . 1 \% )}$ & $\mathbf{7 3 5}(\mathbf{5 0 . 3 \% )}$ & $\mathbf{P}<\mathbf{0 . 0 1}$ \\
\hline
\end{tabular}

Obtained data indicate that normal pregnancy development was significantly more often observed in Group A women, while any kind of unsatisfactory pregnancy outcomes as well as disorders in the health state of the newborns were significantly more often revealed in Group B women.

Calculations of positive predictive value (PPV), sensitivity and specificity of ELI-P-Complex, as well as relative risk of pregnancy complications, adverse pregnancy outcome, and health state of the newborns are presented in Table 5.

Positive predictive value (PPV) of ELI-P-Complex for prediction of pregnancy complication:

$$
\text { PPV }=\frac{\text { True Positive }}{\text { True Positive }+ \text { False Positive }} \times 100 \%
$$

True Positive-Women from 2 subgroups with pregnancy complications.

False Positive-Women from 2 subgroups without pregnancy complications.

$$
\mathrm{PPV}=\frac{257+1763}{(257+1763)+(28+105)} \times 100 \%=93.82 \%(95 \% \mathrm{CI}: 92.72 \% \text { to } 94.80 \%)
$$

Sensitivity and Specificity of ELI-P-Complex for prediction of pregnancy complication:

$$
\text { Sensitivity }=\frac{\text { True Positive }}{\text { True Positive }+ \text { False Negative }} \times 100 \%
$$

True Positive-Women from 2 subgroups with pregnancy complications.

False Negative-Women from 1 subgroup with pregnancy complications.

$$
\text { Sensitivity }=\frac{257+1763}{(257+1763)+148} \times 100 \%=93.17 \%(95 \% \mathrm{CI}: 92.03 \% \text { to } 94.20 \%)
$$


Table 5. Positive predictive value (ppv), sensitivity, specificity and relative risk (rr) calculation.

\begin{tabular}{|c|c|c|}
\hline \multicolumn{3}{|c|}{ Positive predictive value (PPV), sensitivity and specificity } \\
\hline $\begin{array}{l}\text { Group 1. Women with deviations of immune reactivity in one or more parameters of } \\
\text { ELI-P-Complex }(n=2153)\end{array}$ & $257+1763$ & 133 \\
\hline Group 2. Women with normal results of ELI-P-Complex $(\mathrm{n}=1816)$ & 148 & $1403+145+120$ \\
\hline \multicolumn{3}{|c|}{ Relative risk (RR) for pregnancy complications } \\
\hline $\begin{array}{l}\text { Group 1. Women with deviations of immune reactivity in one or more parameters of } \\
\text { ELI-P-Complex }(\mathrm{n}=2153)\end{array}$ & $257+1763$ & $28+105$ \\
\hline Group 2. Women with normal results of ELI-P-Complex $(n=1816)$ & $127+11+8$ & $1403+145+122$ \\
\hline \multicolumn{3}{|c|}{ Relative risk (RR) for adverse pregnancy outcome } \\
\hline $\begin{array}{l}\text { Group 1. Women with deviations of immune reactivity in one or more parameters of } \\
\text { ELI-P-Complex }(n=2153)\end{array}$ & $120+753$ & $165+1115$ \\
\hline Group 2. Women with normal results of ELI-P-Complex $(n=1816)$ & $108+9+8$ & $1422+147+122$ \\
\hline \multicolumn{3}{|c|}{ Relative risk (RR) for newborns health state } \\
\hline $\begin{array}{l}\text { Group 1. Women with deviations of immune reactivity in one or more parameters of } \\
\text { ELI-P-Complex }(\mathrm{n}=1549)\end{array}$ & $714+109$ & $620+106$ \\
\hline Group 2. Women with normal results of ELI-P-Complex $(n=1663)$ & $87+12+12$ & $1297+140+115$ \\
\hline
\end{tabular}

$$
\text { Specificity }=\frac{\text { True Negative }}{\text { False Positive }+ \text { True Negative }} \times 100 \%
$$

True Negative-Women from 3 subgroups without pregnancy complications.

False Positive-Women from 1 subgroup without pregnancy complications.

$$
\text { Specificity }=\frac{1403+145+120}{133+(1403+145+120)} \times 100 \%=92.62 \%(95 \% \text { CI }: 91.31 \% \text { to } 93.78 \%)
$$

Relative Risk (RR) for pregnancy complications

$$
\mathrm{RR}=\frac{a /(a+b)}{c /(c+d)}=\frac{2020 /(2020+133)}{146 /(146+1670)}=11.67(95 \% \mathrm{CI}: 9.9851 \text { to } 13.6392 ; \mathrm{P}<0.0001)
$$

a-Positive (bad) outcome (Group 1), b-Negative (good) outcome (Group 1), c-Positive (bad) outcome (Group 2), d-Negative (good) outcome (Group 2).

Relative Risk (RR) for adverse pregnancy outcome

$$
\mathrm{RR}=\frac{a /(a+b)}{c /(c+d)}=\frac{873 /(873+1280)}{125 /(125+1691)}=5.8908(95 \% \mathrm{CI}: 4.9365 \text { to } 7.0296 ; \mathrm{P}<0.0001)
$$

a-Positive (bad) outcome (Group 1), b-Negative (good) outcome (Group 1), c-Positive (bad) outcome (Group 2), d-Negative (good) outcome (Group 2).

Relative Risk (RR) for newborns' health state

$$
\mathrm{RR}=\frac{a /(a+b)}{c /(c+d)}=\frac{823 /(823+726)}{111 /(111+1552)}=7.9601(95 \% \mathrm{CI}: 6.6110 \text { to } 9.5845 ; \mathrm{P}<0.0001)
$$

a-Positive (bad) outcome (Group 1), b-Negative (good) outcome (Group 1), c-Positive (bad) outcome (Group 2), d-Negative (good) outcome (Group 2).

\section{Discussion}

Diagnostics with ELI-P-complex test can only be understood clearly realizing that auto-antibodies are always present in the body of any healthy person, and their values vary within certain normal ranges [18] [23] [24]. Persistent abnormal serum content of auto-antibodies indicates the presence of a pathological process in a certain 
organ. This applies to a woman preparing to become a mother as well. Almost all processes taking place in her body affect the state of her immune system. Induced immune changes appear clearly by deviations in production of cytokines and antibodies, and may be a leading cause of adverse outcomes of pregnancy (miscarriages, inborn anomalies, etc.) [18] [21] [25].

Our study, as well as the previous research [11] [12] [15] [16] [23], has demonstrated that infectious inflammatory processes of various etiology, endocrine changes, antiphospholipid syndrome, and other disorders in the female body, as well as combinations of the above mentioned changes that have causal relationship to adverse pregnancy outcomes are accompanied by certain abnormalities in the production of embryotropic antibodies. During the preconception care after the first ELI-P-Complex testing, abnormal content of embryotropic antibodies was revealed in 92.4\% $(\mathrm{n}=2041)$ of Group A women. Such disorders $(\mathrm{P}>0.05)$ were revealed in Group B as well during the first trimester of pregnancy in $93.5 \%(n=1868)$ of women.

Provided that main etiologic factors impairing the function of female reproductive system are eliminated, complete normalization or significant improvement of the immune reactivity parameters of the embryotropic antibodies system is observed [11] [12] [23]. In our case, normalization or significant improvement of immune reactivity was reported in 1692 (82.9\%) women from Group A after preconception care. It correlated with satisfactory clinical data, normalization of the menstrual cycle, and laboratory data. In 349 (17.1\%) women, improvement in parameters of immune reactivity was not achieved for various reasons within the 4-month period specified in the study protocol.

Earlier [26] it was demonstrated that effective elimination of major etiologic factors (infectious inflammatory process, endocrine disorder, etc.) is associated with normalization of synthesis of the embryotropic antibodies and results in 5- to 8-fold decrease in the incidence of adverse pregnancy outcomes. In our study, comparative analysis of the pregnancy course in women from the studied groups has shown that preconception care using ELI-P-Complex test significantly (up to 12 times $(\mathrm{RR}=11.67)$ ) decreases the number of complications throughout the whole gestational period as compared to the patients who did not undergo ELI-P-Complex testing prior to pregnancy. Furthermore, even the most thorough follow-up of the patients from Group B and treatment of complications occurring throughout the pregnancy did not always provide the desired effect, which negatively affected some pregnancy outcomes (761 (38.1\%) cases of adverse pregnancy outcomes). Recurrent adverse pregnancy outcomes in a majority of cases were observed in patients with significantly impaired production of one or more regulatory antibodies: the level of serum antibodies was lower than $-60 \mathrm{CU}$ or higher than $+40 \mathrm{CU}$ according to the results of ELI-P-Complex test.

Not only immune activation but immune suppression as well can lead to pregnancy disorders. Thus, according to Makarov and Osipova [27] immune suppression related to a steady decrease in the serum content of some embryotropic a-Abs is a marker of future preeclampsia. Preeclampsia in our study was observed in $84 \%$ of women with immune suppression.

Violations of intrauterine development conditions caused by changes in production of maternal a-Abs are associated with the death of the embryo or fetus (although not in all cases); however, they almost always negatively affect the child's health state. In accordance to observations by Kluchnikov and Zamaleeva, if children were born to women with the normal parameters of ELI-P-Complex method, at least $70 \%$ of them were evaluated as practically healthy at ages $4-6$ years old. On the contrary, no more than $15 \%$ children were evaluated as practically healthy if they were born to women with steadily deviated parameters of serum a-Abs [11] [28]. As our study has demonstrated, impaired production of embryotropic antibodies negatively affects development of the embryo and fetus and results in increased frequency of death, congenital defects or unsatisfactory health state. Only $50.3 \%(n=735)$ of the newborns out of all children born in Group B were completely healthy, while $88.1 \%(\mathrm{n}=1543)$ of the children from Group A were born absolutely healthy.

It should be noted that a threatened pregnancy loss was observed in $1746(87.4 \%)$ patients from Group B in the first trimester of pregnancy and in 957 (47.9\%) patients during the second trimester; thus, $1660(83.1 \%)$ pregnant women were treated in the in-patient settings. At the same time, threatened pregnancy loss in patients who underwent preconception care (Group A) was observed during the first and second trimesters in 150 (7.6\%) and $71(3.6 \%)$ patients respectively; moreover, only $203(10.3 \%)(\mathrm{P}<0.01)$ of them required hospitalization and received in-patient treatment. This is confirmed by the data from Liza Osipenko study [29] of cost effectiveness of using ELI-P-Complex test in the obstetrical practice.

Finally, it should be noted that impaired ELI-P-Complex test scores, despite the hospitalization and conducted treatment during the whole gestational period, increased the risk of adverse pregnancy outcome by 6 times $(\mathrm{RR}=$ 
5.8908) and the risk of giving birth to an unhealthy child by 8 times $(R R=7.9601)$ as compared to a group of patients with normal ELI-P-Complex test scores.

\section{Conclusion}

In summary, our study has demonstrated that thorough preconception care using ELI-P-Complex test decreases the risk of recurrent adverse pregnancy outcome. Assessment of serum embryotropic antibodies content with ELI-P-Complex test may serve as an objective criterion of female readiness to give birth to a healthy child as well as a criterion of efficacy and sufficiency of therapeutic measures conducted throughout the period of preconception care in women with a history of adverse pregnancy outcomes. Persistent changes in the antibodies against proteins (regulators of embryogenesis) must be considered an alert signal from the body of a future mother. We believe that ELI-P-Complex test may be recommended for a wide use during the preconception care of women with a history of adverse pregnancy outcomes.

\section{References}

[1] NICE (2012) Clinical Knowledge Summaries, Pre-Conception-Advice and Management.

http://cks.nice.org.uk/pre-conception-advice-and-management\#!scenariobasis:11

[2] The March of Dimes Data. http://www.amputee-coalition.org/inmotion/jan feb 06/congenital limb part1.html

[3] Stirrat, G.M. (1990) Recurrent Miscarriage. The Lancet, 336, 673-675. http://dx.doi.org/10.1016/0140-6736(90)92159-F

[4] Regan, L., Braude, P. and Trembath, P. (1989) Influence of Past Reproductive Performance on Risk of Spontaneous Abortion. BMJ, 299, 541-545. http://dx.doi.org/10.1136/bmj.299.6698.541

[5] Greenwood, R., Samms-Vaughan, M., Golding, J. and Ashley, D. (1994) Past Obstetric History and Risk of Perinatal Death in Jamaica. Paediatric and Perinatal Epidemiology, 8, 40-53. http://dx.doi.org/10.1111/j.1365-3016.1994.tb00490.x

[6] Samueloff, A., Xenakis, E.M., Berkus, M.D., Huff, R.W. and Langer, O. (1993) Recurrent Stillbirth: Significance and Characteristics. The Journal of Reproductive Medicine, 38, 883-886.

[7] Reddy, U.M. (2007) Prediction and Prevention of Recurrent Stillbirth. Obstetrics \& Gynecology, 110, 1151-1164. http://dx.doi.org/10.1097/01.AOG.0000287616.71602.d0

[8] Radhupathy, R. (1997) Th1-Type Immunity Is Incompatible with Successful Pregnancy. Immunology Today, 18, 478451. http://dx.doi.org/10.1016/S0167-5699(97)01127-4

[9] Poletaev, A.B. (2008) Immunophysiology and Immunopathology. MIA Press, Moscow.

[10] Sukhih, G.T. and Van'ko, L.V. (2003) Immunology of Pregnancy. Russian Academy of Medical Sciences Publisher, Moscow.

[11] Kliuchnikov, C.O., Poletaev, A.B., Budikina, T.S. and Generalova, G.A. (2001) New Immune Biotechnologies in Neonatology and Paediatrics. In: Demin, S.F. and Kliuchnikov, C.O., Eds., Lectures in Paediatrics: Pathologies in Newborns and Infants, Volume 1, RGMU, Moscow, 243-267.

[12] Budykina, T.C. and Poletaev, A.B. (2002) Methodology for Evaluation of Fetal Pathological Development. RF Patent No. 2208791.

[13] Kalsi, J. and Isenberg, D.A. (2000) Measurement of Natural Autoantibodies. In: Shoenfeld, Y. and Isenberg, D.A., Eds., Natural Autoantibodies, CRS Press, Boca Raton, 35-57.

[14] Poletaev, A.B. and Morozov, S.G. (2002) Part I. Natural Autoantibodies. In: Poletaev, A.B., Morozov, S.G. and Kovaliov, I.E., Eds., The Regulatory Metasystem: Immuno-Neuro-Endocrine Regulation of General Homeostasis, Meditsina, Moscow, 5-162.

[15] Marai, I., Carp, H., Shai, S., Shabo, R., Fishman, G. and Shoenfeld, Y. (2004) Autoantibody Panel Screening in Recurrent Miscarriages. American Journal of Reproductive Immunology, 51, 235-240. http://dx.doi.org/10.1111/j.1600-0897.2004.00153.X

[16] Shoenfeld, Y., Carp, H.J., Molina, V., Blank, M., Cervera, R., Balasch, J., Tincani, A., Faden, D., Lojacono, A., Doria, A., Konova, E. and Meroni, P.L. (2006) Autoantibodies and Prediction of Reproductive Failure. American Journal of Reproductive Immunology, 56, 337-344. http://dx.doi.org/10.1111/j.1600-0897.2006.00434.x

[17] Cohen, J., Bakimer, R. and Shoenfeld, Y. (1995) Fecundity in Autoimmune Diseases. Early Pregnancy, 1, 93-105.

[18] Poletaev, A.B. (2010) Physiological Immunology. Micklosh Publishers, Moscow.

[19] Poletaev, A. (2013) The Main Principles of Adaptive Immune System Function: Self-Recognition, Self-Interaction, 
and Self-Maintenance. In: Poletaev, A.B., Ed., Physiologic Autoimmunity and Preventive Medicine, Bentham Science Publishers, Sharjah, Oak Park and Bussum, 3-20. http://dx.doi.org/10.2174/9781608057245113010004

[20] Niukhnin, M.A. (2007) Autoantibodies Analysis and the Problem of Optimization of the Tactics of the Pregnant Women Treatment. PhD Dissertation, Kazan State Medical Academy, Kazan.

[21] Poletaev, A. (2012) Maternal Immunity, Pregnancy and Child's Health. In: Sifakis, S. and Vrachnis, N., Eds., From Preconception to Postpartum, InTech, Rijeka, 41-56.

[22] Grabar, P. (1968) About Autoantibodies. In: Ado, A.D., Ed., Problems of Reactivity in Pathology, Meditsina, Moscow, 35-52.

[23] Poletaev, A.B., Maltseva, L.I., Zamaleeva, R.S., Nukhnin, M.A. and Osipenko, L.G. (2007) Application of ELI-PComplex Method in Clinical Obstetrics. American Journal of Reproductive Immunology, 57, 294-301. http://dx.doi.org/10.1111/j.1600-0897.2007.00479.x

[24] Tauber, A.I. (2014) Reconceiving Autoimmunity: An Overview. Journal of Theoretical Biology, 375, 52-60.

[25] Poletaev, A., Poletaeva, A., Pukhalenko, A., Zamaleeva, R., Cherepanova, N. and Frizin, D. (2014) Adaptive Maternal Immune Deviations as a Ground for Autism Spectrum Disorders Development in the Child. Folia Medica, 56, 73-80. http://dx.doi.org/10.2478/folmed-2014-0011

[26] Serova, O.F. (2000) Pre-Gestational Treatment of the Women with Habitual Miscarriages. MD Dissertation, People's Friendship University of Russia (RUDN), Moscow.

[27] Makarov, O.V., Osipova, N.A. and Poletaev, A.B. (2009) Autoantibodies and Diagnosis of Pre-Eclampsy. MedicineXXI Century, 14, 28-32.

[28] Zamaleeva, R.S., Mal'tseva, L.I., Cherepanova, N.A., Bukatina, S.V. and Nyukhnin, M.A. (2009) Clinical Value of Definition of a Level of Regulatory Autoantibodies for an Estimation of Risk of Development of Gestosis. Prakticheskaya Meditsina, 34, 68-71.

[29] Osipenko, L. (2005) System Dynamics in Early Health Technology Assessment: Prenatal Screening Technology. PhD Dissertation, Stevens Institute of Technology, Hoboken. 